\title{
A Librarian, a Politician, a UX Expert, and a Cyberbully Walk into a Special Issue
}

In this special issue, we present four selected papers from the 2018 Hawaii International Conference on System Sciences (HICSS) conference, which took place in the Big Island of Hawaii. Fifteen conference papers were revised, extended, and then submitted for consideration to the ACM TSC special issue's fast track. The original conference papers were revised based on the conference feedback and were significantly extended in scope. After a thorough process of peer review and revisions, the four papers that were selected are: "Social Media Ethics: A Rawlsian Approach to Hypertargeting and Psychometrics in Political and Commercial Campaigns" by Morten Bay, "A Socio-contextual Approach in Automated Detection of Public Cyberbullying on Twitter" by Nargess Tahmasbi and Elham Rastegari, "A Participation Architecture to Support User Peripheral Participation in a Hybrid FOSS Community” by Susan Gasson and Michelle Purcell, and "Understanding Social Roles in an Online Community of Volatile Practice: A Study of User Experience Practitioners on Reddit" by Yubo Kou, Colin Gray, Austin Toombs, and Robin Adams. The four papers in this special issue examine diverse aspects of social computing, from its impacts on political campaigns to the automated detection of cyberbullying and the dynamics of online communities.

The paper that opens this special issue of TSC discusses the ethics of using hypertargeting and psychometrics in political campaigns. This applied ethics paper by Bay begins with a review of the uses of hypertargeting and psychometrics in social media and of the scant literature on the ethics of using these marketing methods. After introducing Rawlsian ethics and principles, the author carefully discusses the ethics of moving these digital marketing methods from the context of commercial marketing to that of political marketing. This discussion uses evidence from two cases from the 2016 US presidential elections: the Cambridge Analytica/Facebook scandal and the very close margin $(0.2 \%)$ that determined the election results in Michigan. The paper concludes that, within the Rawlsian framework, using such marketing methods for political advertising violates Rawlsian ethics and should be regulated.

Cyberbullying is another significant social computing issue that calls for our attention and solution. In the subsequent paper, Tahmasbi and Rastegari tackle the challenge of automatic detection of public cyberbullying by proposing a more sophisticated approach to detecting cyberbullying, which goes beyond the content of the message by examining meta-components. This novel approach allows the two researchers to examine not only textual features of Twitter messages but also network features and Tweet metadata. This approach is tested on two cases of Twitter-based public cyberbullying. The findings show that adding these socio-contextual features to the classification model significantly increases the accuracy, precision, and recall of the classification model. They also demonstrate that cyberbullying texts do not necessarily use highly negative and profane language. This line of investigation promises to diversify the toolkit of methods available for cyberbullying detection.

The paper by Gasson and Purcell examines a free open-source software (FOSS) community that develops and maintains an open-source software product that supports libraries. Focusing on how non-technical users participate in online communities that were originally designed around

2018 Copyright is held by the owner/author(s).

2469-7818/2018/12-ART14e

https://doi.org/10.1145/3293613 
assumptions of technical participation, the researchers reveal the critical roles played by these non-technical peripheral participants in the success of the project. Using three qualitative methods-community ethnography, trace ethnography, and analysis of technology platform affordances-the researchers explore how these peripheral participants were involved in the community, and how the socio-technical affordances of the supporting infrastructure constrain and enable their participation. The researchers conclude that the technical affordances of a typical FOSS community participation architecture did not sufficiently mediate the participation of these peripheral non-technical users and that the meaningful participation was mediated by boundary spanners who took on knowledge-brokering and organizational bridging roles. Thus, the successful innovative outcomes of this FOSS community were the result of a combination of technical and social affordances that effectively linked the peripheral participants to the core activities of the community. Clear implications for practice follow from this work.

In the concluding paper of the issue, Kou, Gray, Toombs, and Adams also researched an online community of practice-a Reddit user experience (UX) community where practitioners socialize and learn-but the researchers focused on a unique aspect of this community: the highly volatile and rapidly evolving nature of UX practice. In particular, the researcher team sought to understand how this volatility influence participants' roles in the UX community and how these different roles influence communication and interaction patterns. The research identified five social roles of community members, and it validated and characterized the social roles using linguistic analysis, social network analysis, and visualization techniques. This rich and insightful description informs our understanding of the social and technological aspects of the design of communities of practice, as well as of UX practice and of UX pedagogy.

The four papers in this special issue emphasize the social impact of social computing research. They not only innovate theory but also shed new light on the impact of processes that take place in online social spaces. We see how these processes influence the ability of community members to achieve their goals; how they affect local, national, and world politics; and how they influence bullying that threatens the sense of safety of individuals. Taken together, these papers demonstrate the use and innovative application of quantitative, qualitative, mixed, and philosophical methods to improve our understanding of social computing and its impact on our everyday life.

Kevin Crowston

Editor-in-Chief

Xuefei (Nancy) Deng

Yoram M. Kalman

Guest Editors 